\title{
Does postural awareness contribute to exercise-induced improvements in neck pain intensity? A secondary analysis of a randomized controlled trial evaluating Tai Chi and neck exercises
}

Lauche, Romy ; Wayne, Peter M ; Fehr, Johannes ; Stumpe, Christoph ; Dobos, Gustav ; Cramer, Holger

\begin{abstract}
STUDY DESIGN: Secondary analysis of a randomized controlled trial. OBJECTIVE: This secondary analysis aims to examine associations of improvement of chronic neck pain with patients' and intervention-related characteristics. SUMMARY OF BACKGROUND DATA: Previous research has found that Tai Chi and neck exercises significantly improved chronic nonspecific neck pain; however, the factors for treatment success remain unclear. METHODS: Subjects with chronic nonspecific neck pain were randomly assigned to 12 weeks of group Tai Chi or conventional neck exercises, and they attended 12 weekly sessions of 60 to 90 minutes. The interventions included exercises to improve body awareness, that is, interoceptive and postural awareness. A linear forward stepwise regression analysis was conducted to examine associations with improvements in neck pain intensity. Potential predictor variables included baseline pain, age, sex, the type of intervention, attendance rate and home practice duration, and changes in psychological well-being, perceived stress, and postural and interoceptive awareness during the study. RESULTS: Overall 75 patients were randomized into Tai Chi or conventional exercises, with the majority being women (78.7\%). Participants reported an average pain intensity of $50.7 \pm 20.4 \mathrm{~mm}$ visual analog scale at baseline, and the average reduction of pain intensity in both groups was $21.4 \pm 21.3$ mm visual analog scale. Regression analysis revealed that reductions in pain intensity from baseline to 12 weeks were predicted by higher pain intensity at baseline $(\mathrm{r}=0.226, \mathrm{P}<0.001)$, a decrease in anxiety $(\mathrm{r}=$ $0.102, \mathrm{P}=0.001)$, and an increase in postural awareness $(\mathrm{r}=0.078, \mathrm{P}=0.0033)$, explaining a total of $40.6 \%$ of variance. CONCLUSION: Neck pain improvement was significantly associated with changes in postural awareness in subject with chronic nonspecific neck pain independent of treatment characteristics. Training of postural awareness might be an important mechanism of action of different exercise-based interventions for chronic neck pain. LEVEL OF EVIDENCE: N / A.
\end{abstract}

DOI: https://doi.org/10.1097/BRS.0000000000002078

Posted at the Zurich Open Repository and Archive, University of Zurich

ZORA URL: https://doi.org/10.5167/uzh-142693

Journal Article

Published Version

Originally published at:

Lauche, Romy; Wayne, Peter M; Fehr, Johannes; Stumpe, Christoph; Dobos, Gustav; Cramer, Holger (2017). Does postural awareness contribute to exercise-induced improvements in neck pain intensity? A secondary analysis of a randomized controlled trial evaluating Tai Chi and neck exercises. Spine, 42(16):1195-1200. 
DOI: https://doi.org/10.1097/BRS.0000000000002078 


\title{
Does Postural Awareness Contribute to Exercise- Induced Improvements in Neck Pain Intensity? A Secondary Analysis of a Randomized Controlled Trial Evaluating Tai Chi and Neck Exercises
}

\author{
Romy Lauche, PhD, ${ }^{*} \dagger$ Peter M. Wayne, PhD ${ }^{\ddagger}{ }^{\ddagger}$ Johannes Fehr, ${ }^{*}$ Christoph Stumpe, MSc, ${ }^{*}$ \\ Gustav Dobos, MD, PhD, ${ }^{*}$ and Holger Cramer, $\mathrm{PhD}^{*, \dagger}$
}

\begin{abstract}
Study Design. Secondary analysis of a randomized controlled trial.

Objective. This secondary analysis aims to examine associations of improvement of chronic neck pain with patients' and intervention-related characteristics.
\end{abstract}

Summary of Background Data. Previous research has found that Tai Chi and neck exercises significantly improved chronic nonspecific neck pain; however, the factors for treatment success remain unclear.

Methods. Subjects with chronic nonspecific neck pain were randomly assigned to 12 weeks of group Tai Chi or conventional neck exercises, and they attended 12 weekly sessions of 60 to 90 minutes. The interventions included exercises to improve body awareness, that is, interoceptive and postural awareness. A linear forward stepwise regression analysis was conducted to examine associations with improvements in neck pain intensity. Potential predictor variables included baseline pain, age, sex, the type of intervention, attendance rate and home practice duration, and changes in psychological well-being, perceived

From the *Department of Internal and Integrative Medicine, Kliniken EssenMitte, Faculty of Medicine, University of Duisburg-Essen, Essen, Germany; ${ }^{\dagger}$ Australian Research Centre in Complementary and Integrative Medicine (ARCCIM), University of Technology Sydney, Sydney, Australia; and ${ }^{\ddagger}$ Osher Center for Integrative Medicine, Brigham and Women's Hospital and Harvard Medical School, Boston, MA.

Acknowledgment date: August 25, 2016. First revision date: November 1, 2016. Acceptance date: December 12, 2016.

Johannes Fehr and Christoph Stumpe have contributed equally to the project.

The manuscript submitted does not contain information about medical device(s)/drug(s).

No funds were received in support of this work.

No relevant financial activities outside the submitted work.

Address correspondence and reprint requests to Romy Lauche, PhD, Australian Research Centre in Complementary and Integrative Medicine (ARCCIM), University of Technology Sydney, Faculty of Health, Level 8, BIdg 10,

235-253 Jones St, Ultimo NSW 2007, Australia;

E-mail: romy.lauche@uts.edu.au

DOI: 10.1097/BRS.0000000000002078 stress, and postural and interoceptive awareness during the study.

Results. Overall 75 patients were randomized into Tai Chi or conventional exercises, with the majority being women $(78.7 \%)$. Participants reported an average pain intensity of $50.7 \pm 20.4$ $\mathrm{mm}$ visual analog scale at baseline, and the average reduction of pain intensity in both groups was $21.4 \pm 21.3 \mathrm{~mm}$ visual analog scale. Regression analysis revealed that reductions in pain intensity from baseline to 12 weeks were predicted by higher pain intensity at baseline $\left(r^{2}=0.226, P<0.001\right)$, a decrease in anxiety $\left(r^{2}=0.102, P=0.001\right)$, and an increase in postural awareness $\left(r^{2}=0.078, P=0.0033\right)$, explaining a total of $40.6 \%$ of variance.

Conclusion. Neck pain improvement was significantly associated with changes in postural awareness in subject with chronic nonspecific neck pain independent of treatment characteristics. Training of postural awareness might be an important mechanism of action of different exercise-based interventions for chronic neck pain.

Key words: body awareness, neck exercises, neck pain, postural control, Tai Chi

Level of Evidence: N/A

Spine 2017;42:1195-1200

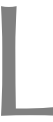

arge bodies of publications have investigated the efficacy and safety of exercise interventions for treating chronic neck and back pain. ${ }^{1-3}$ While most clinical trials are focused on the outcomes such as pain intensity or physical function, less is known about factors contributing to the observed treatment effect. Identifying predictive variables, however, could help to improve the design and implementation of clinical interventions, and to better match patients with potentially effective interventions.

Among the variables that other exercise studies have evaluated for predicting response are participant age, sex, and disability, and the type or dosage of interventions. ${ }^{4-6}$ 
Improvements in body awareness might also be highly relevant to chronic neck and back pain, because studies have repeatedly pointed at the link between those pain conditions and disturbed body image, especially in chronic back $^{7-9}$ and neck pain. ${ }^{10,11} \mathrm{~A}$ variety of body image measures have been used in research before including measures of proprioception and kinesthetic sense, ${ }^{12-16}$ of interoceptive awareness, ${ }^{17,18}$ and of postural awareness. ${ }^{19}$ Targeting body awareness is assumed to improve chronic pain and function.

A recent clinical trial on the effects of Tai Chi and neck exercises in chronic neck pain has been published, and results indicate not only significant improvements in pain intensity, but also changes in postural awareness; their interplay, however, had not been investigated. Thus the aim of this secondary analysis is to determine factors associated with treatment success in this trial.

\section{MATERIALS AND METHODS}

This is a secondary analysis of a randomized controlled trial on the effects of Tai Chi and neck exercises on chronic nonspecific neck pain. A detailed description of the methodology and results can be found elsewhere. ${ }^{19}$ Briefly, the trial was conducted in 2014 and 2015 in the Department of Internal and Integrative Medicine in Essen, Germany. The study was approved by the ethics committee of the University Hospital Essen (approval number: 13-5672-BO) and registered at ClinicalTrials.gov (registry number: NCT02222051), before subject recruitment. The trial was three-armed, and Tai Chi was compared with a wait list control group and an active control intervention (neck exercises). Both Tai Chi and neck exercises were held in groups with 10 to 15 participants per class each, and subjects met once weekly for 60 to 90 minutes for a total of 12 weeks. Participants were also asked to practice outside of classes for at least 15 minutes each day.

The sample included adult subjects with at least moderate chronic nonspecific neck pain without major medical conditions or comorbidities, invasive treatments in the recent past or during the trial, or regular Tai Chi or yoga practice in the past 6 months. For more details on inclusion and exclusion criteria please see the published trial. ${ }^{19}$ The subjects were randomly assigned to their groups, and outcomes were assessed at baseline and after 12 weeks.

\section{Interventions}

The Tai Chi intervention was based on the 13 forms from Mantak Chia, ${ }^{20}$ a variation of the internationally recognized Yang style Tai Chi; and it was practiced according to the official training manual. The neck exercise training was similar to those taught in rehabilitation programs, with exercises and education for a healthy back. In addition to isometric and dynamic mobilization, stretching and strengthening neck and core exercises weekly classes also included basic training of ergonomic principles.

Both interventions also aimed to improve participants' body awareness by incorporating a variety of proprioceptive (and interoceptive) exercises into the classes. Supported by breathing exercises, and guided imagery participants were instructed to perceive their posture, to actively change it, and to observe any changes as a result of the exercises. The exercises aimed to increase body awareness in everyday life as a means to reduce neck pain, and neck pain-related disability.

\section{Outcomes}

Outcomes were measured at baseline and at week 12, that is, at the end of the study intervention. At the baseline assessment all patients rated their expectations that Tai Chi or neck exercises would be able to improve their neck pain on a 0 to 10 Numerical Rating Scale with 10 indicating "highest possible expectation."

Pain intensity was measured using a 0 to $100 \mathrm{~mm}$ visual analog scale (VAS) from the German Pain Questionnaire 21,22 with $0 \mathrm{~mm}$ indicating "no neck pain at all" and $100 \mathrm{~mm}$ indicating "worst neck pain imaginable."

Psychological distress was measured by the Hospital Anxiety and Depression Scale ${ }^{23,24}$ with the two dimensions anxiety and depression, with higher values indicating higher psychological distress.

The degree to which patients perceived their lives as stressful was measured using the German Version of the Perceived Stress Scale (PSS). ${ }^{25,26}$ The PSS consists of 10 items asking how often subjects find their lives unpredictable, uncontrollable, and overloaded in the last month; with higher scores being indicative of higher perceived stress in life.

Interoception, that is, the sensitivity toward stimuli originating from within the body, was measured using the Multidimensional Assessment of Interoceptive Awareness instrument (MAIA) ${ }^{17}$ which consists of 40 items resulting in eight separate dimensions of interoceptive awareness; and higher scores each represent higher awareness.

Postural awareness was measured using the Postural Awareness Scale (PAS) (Manuscript in preparation) with 12 items that describe the awareness of body posture and postural control. The 12 -item instrument consists of two scales with six items each, and it has shown good psychometric properties. The scale Effortless Awareness and Connectedness describes high levels of postural awareness; the subject regularly becomes aware of their posture, and being aware requires only low efforts. This scale also describes a connectedness between body and mind, that is, a sense of the relationship between posture and well-being. The scale Forced Awareness and Detachment on the contrary indicates low levels of postural awareness, which and a substantial amount of attention and focus is necessary to become aware of the bodily posture, and only pain or discomfort will remind participants of dysfunctional posture. Higher scores on both scales are indicative of higher awareness and connectedness (factor 1), and forced awareness and detachment (factor 2). The instrument can be found in the supplemental table (http://links.lww.com/ $\mathrm{BRS} / \mathrm{B} 252)$. 
Attendance rate was measured using a record of attendance in each class, and the duration of home practice was calculated from the daily logs on which participants recorded their daily home practice in minutes.

\section{Experiences With the Interventions}

Participants who participated in Tai Chi and neck exercises were asked to provide short feedback on their experiences with the respective interventions, with a special focus on body awareness. Semistandardized mini-interviews were taped, transcribed verbatim and analyzed using content analysis, ${ }^{27}$ and results were paraphrased. Some statements were translated into English for illustration purpose.

\section{Statistical Analysis}

All quantitative analyses were based on the intention to treat population, that is, each patient providing baseline data was included in the final analysis. Missing data were completed using the Markov chain Monte Carlo multiple imputation method in SPSS. A set of 50 imputations was generated, and the arithmetic mean of those imputations was used for the analysis.

To analyze the effect of changes in the Perceived Stress Scale (PSS), the Multidimensional Assessment of Interoceptive Awareness (MAIA), and the Postural Awareness Scale (PAS) on changes in pain intensity a linear forward stepwise regression analysis with linear or dichotomous predictor variables was conducted for all parameters that were significantly correlated with changes in pain intensity. To control for possible effects of sociodemographic variables, age and sex were included in regression analyses in addition to the number of attended classes and duration of home practice. Significance level was set at $\alpha=0.05$; and changes in $R^{2}$ were reported for each variable included in the model.

All analyses were performed using the Statistical Package for Social Sciences software (IBM SPSS Statistics for Windows, release 22.0, IBM Corp, Armonk, NY).

\section{RESULTS}

Altogether 75 of 114 subjects in the trial were randomized to Tai Chi or neck exercises, and the majority of them were women $(78.7 \%)$. Participants reported an average pain intensity of $50.7 \pm 20.4 \mathrm{~mm}$ VAS at baseline, and the average reduction of pain intensity in both groups was $21.4 \pm 21.3 \mathrm{~mm}$ VAS.
Regression analysis revealed that reductions in pain intensity from baseline to 12 weeks were associated with higher pain intensity at baseline $\left(r^{2}=0.226, P<0.001\right)$, a decrease in anxiety (Hospital Anxiety and Depression Scale) $\left(r^{2}=0.102, P=0.001\right)$, and an increase in the Effortless Awareness and Connectedness score from the Postural Awareness Scale $\left(r^{2}=0.078, P=0.0033\right)$, explaining a total of $40.6 \%$ of variance $(P<0.0001$; Table 1$)$. No other variables were associated with pain reduction.

Qualitative data revealed that when asked about their body awareness, and potential changes due to Tai Chi or neck exercises, participants reported that both the interventions had changed the ability to perceive the body, including posture ("I am certain that it changed lastingly, that [since the intervention] I am more aware of my posture, especially during working in a sitting position." Subject 87). Participants indicated that awareness has widened, and expanded to areas of the body that had gone unnoticed before ("It has changed in a way, that the awareness has expanded to the neck and the head." Subject 39). They also reported that their focus more often turned toward their posture and their movements, as compared to before the intervention. Becoming more focused on body awareness was mostly described as an active process; and participants reminded themselves to examine themselves while standing, sitting, or walking. Some participants also, however, described a sudden, almost automatic awareness of posture at some times (" ... and now and then it [posture] just comes to my mind, when I am walking on the office floor." Subject 32). The increased postural awareness also led to changes in posture and habitual movement pattern, such as sitting straight or walking more upright; and patients felt that they could actively affect their pain (and well-being) by observing and modifying their postural and movement patterns ("When I am having a moment of time, or when I am working in a sitting position, then I might become aware, I sit up straight, take a deep breath, relax my belly to breath freely, those things have changed." Subject 112).

\section{DISCUSSION}

It was previously reported that Tai Chi and neck exercises led to a significant decrease in pain intensity in participants with chronic nonspecific neck pain. ${ }^{19}$ This secondary analysis informs these findings in ascertaining that the reduction in pain intensity was associated with several

\begin{tabular}{|l|c|c|c|c|}
\hline TABLE 1. Predictors for Changes in Pain Intensity As per Regression Analysis \\
\hline Predictor & $\boldsymbol{R}^{\mathbf{2}}$ & Change $\boldsymbol{R}^{\mathbf{2}}$ & Beta $\mathbf{( 9 5} \% \mathbf{C I})$ & $\boldsymbol{P}$ \\
\hline Pain at baseline (VAS) & 0.226 & 0.226 & $-0.458(-0.652 ;-0.264)$ & $<0.001$ \\
\hline $\begin{array}{l}\text { Difference in anxiety } \\
\text { (HADS) }\end{array}$ & 0.328 & 0.102 & $2.404(0.848 ; 3.960)$ & 0.003 \\
\hline $\begin{array}{l}\text { Change in effortless } \\
\text { awareness and } \\
\text { connectedness }\end{array}$ & 0.406 & 0.078 & $-0.399(-0.659 ;-0.159)$ & 0.003 \\
\hline HADS indicates Hospital Anxiety and Depression Scale; VAS, visual analog scale. \\
\hline
\end{tabular}


factors, including pain intensity at baseline, a decrease in anxiety, and an increase in postural awareness.

The predictor with the highest explained variance was pain intensity at baseline, in that direction that those subjects with higher pain intensity before the trial reporting more improvement than less affected participants. This is a common finding in clinical trials on pain conditions, ${ }^{28,29}$ and it may be related to the fact that there is more potential for improvement in those experiencing more debilitating neck pain compared to participants with minor to moderate pain in the beginning.

Another significant factor is the change in anxiety, that is, a larger improvement in anxiety was associated with a larger improvement in pain intensity. Associations between psychological disorders and musculoskeletal pain have been investigated before, and studies found a higher prevalence of anxiety disorders in chronic pain patients in general, ${ }^{30,31}$ and in chronic neck pain patients. ${ }^{32}$ Although anxiety can be a result of chronic pain rather than a cause and the current analysis can also be interpreted in this direction, it anxiety can aggravate chronic pain by influencing the vegetative nervous system (sympathicotonic increase), which leads to increased muscle tension especially in the neck and shoulder area. ${ }^{33}$ Reducing anxiety may therefore be beneficial for overall psychological well-being, and lead to better outcomes. Of note, the role of anxiety was significant, even though anxiety levels were modest and below thresholds for an anxiety disorder; and only $26.7 \%$ and $16.0 \%$ had scores above the cut-off for potential $(\geq 8)$, and for clinically relevant anxiety disorder $(\geq 11)$, respectively. ${ }^{34}$

Improvements in pain intensity were also predicted by increases in the Effortless Awareness and Connectedness subscale of the Postural Awareness Scale, that is, the more aware participants were of their posture, the less efforts it took them and the more they became aware of the connection between posture and their well-being, the more their pain decreased during the course of the study. Although there is no direct connection between postural awareness and pain, postural awareness may establish the basis for the process of changing habitual postural and movement patterns, that is, postural awareness can be considered a key prerequisite for postural control ${ }^{35}$; and increasing postural awareness should facilitate postural control. At least for Tai Chi, previous research has found compelling evidence for improvements in postural control as indicated by increases in body awareness, ${ }^{36,37}$ improvements of balance and reduced falls, ${ }^{38-40}$ alterations in muscle tone, ${ }^{41}$ and better kinesthetic control. ${ }^{42,43}$ Similar findings have been reported for yoga, as witnessed by improvements in proprioceptive acuity in chronic neck pain ${ }^{44}$ and in postural control in schizophrenia ${ }^{45}$ or older adults. ${ }^{46}$ Of note, although conventional neck exercises might generally not target body awareness to such degree as yoga or Tai Chi, the exercise instructor in the present study was also the Tai Chi instructor; and he incorporated the same amount of body awareness exercises into the exercise class. ${ }^{19}$
Participants in the study mentioned in qualitative interviews that their body awareness had improved; however, what appears to contradict the findings from the regression analysis is that the changes most of them observed seemed to be more a result of active observation rather than an automatic process. There was, however, mention of sudden and unexpected awareness, and that it became much easier to focus one's attention on the bodily posture, which might correspond with the Effortless Awareness and Connectedness subscale of the Postural Awareness Scale.

Interestingly no influence has been found for attendance rate, or home practice duration. Other trials have reported such associations between higher levels yoga practice and short- or long-term improvements in chronic neck or back pain ${ }^{18,29}$; however, the trial duration and sample size in the present study may not have been sufficient. Contrary to yoga which often includes a number of separate static positions, Tai Chi incorporated a series of movements into complex forms, ${ }^{47,48}$ and participants might need more training to reach a proficiency level necessary for significant and sustained changes. It can also be argued that time alone is no sufficient measure for the treatment dosage, and other measures for proficiency are needed. ${ }^{47,48}$

The study has several limitations: First of all the study was planned as a randomized controlled trial to test the effects of Tai Chi and conventional exercises rather than a study to identify predictors for treatment success; therefore, results should be considered preliminary or "hypothesis generating." The sample size for the regression was relatively small, and reduced by a number of dropouts; finally no objective measures of postural awareness were used. The study results further indicate that other yet unknown factors may have contributed to the improvement in neck pain. Further studies may also be conducted to compare the influence of different programs for improving postural awareness.

\section{CONCLUSION}

In addition to psychological well-being, neck pain improvement was significantly associated with postural awareness in subject with chronic nonspecific neck pain independent of treatment characteristics. Training of postural awareness seems to be an important mechanism of action of different exercise-based interventions for chronic nonspecific neck pain. These findings can help tailor-specific exercise interventions for patients with chronic neck pain.

\section{Key Points}

- Improvements in neck pain intensity are associated with decreases in anxiety.

I Improvements in neck pain intensity are associated with changes in postural awareness.

Training of postural awareness might be an important part of exercise-based interventions for pain. 


\section{Acknowledgments}

The authors acknowledge Petra Klose for support during trial development and Jana Hochstein, Lisa Maria Fox, and Britta Seidenstücker for support in data management, and Dr. Jon Wardle for language editing advice.

Supplemental digital content is available for this article. Direct URL citations appearing in the printed text are provided in the HTML and PDF version of this article on the journal's Web site (www.spinejournal.com).

\section{References}

1. Gross A, Kay TM, Paquin JP, et al. Exercises for mechanical neck disorders. Cochrane Database Syst Rev 2015;1:CD004250.

2. Poquet N, Lin CW, Heymans MW, et al. Back schools for acute and subacute non-specific low-back pain. Cochrane Database Syst Rev 2016;4:CD008325.

3. Saragiotto BT, Maher CG, Yamato TP, et al. Motor control exercise for chronic non-specific low-back pain. Cochrane Database Syst Rev (1):2016;CD012004.

4. Baena-Beato PA, Delgado-Fernandez M, Artero EG, et al. Disability predictors in chronic low back pain after aquatic exercise. Am J Phys Med Rehabil 2014;93:615-23.

5. Cecchi F, Negrini S, Pasquini G, et al. Predictors of functional outcome in patients with chronic low back pain undergoing back school, individual physiotherapy or spinal manipulation. Eur $J$ Phys Rehabil Med 2012;48:371-8.

6. Rasmussen-Barr E, Campello M, Arvidsson I, et al. Factors predicting clinical outcome 12 and 36 months after an exercise intervention for recurrent low-back pain. Disabil Rehabil 2012;34:136-44.

7. Levening CG, Hasenbring MI, Kleinert J, Kellmann M. Body image and low back pain. Der Schmerz 2016;30:437-43.

8. Lotze M, Moseley GL. Role of distorted body image in pain. Curr Rheumatol Rep 2007;9:488-96.

9. Flor H. New developments in the understanding and management of persistent pain. Curr Opin Psychiatry 2012;25:109-13.

10. Lauche R, Cramer H, Haller $H$, et al. My back has shrunk: the influence of traditional cupping on body image in patients with chronic non-specific neck pain. Forsch Komplementmed 2012; 19:68-74.

11. Cramer H, Lauche R, Haller H, et al. I'm more in balance": a qualitative study of yoga for patients with chronic neck pain. I Altern Complement Med 2013;19:536-42.

12. Kerr CE, Shaw JR, Wasserman RH, et al. Tactile acuity in experienced Tai Chi practitioners: evidence for use dependent plasticity as an effect of sensory-attentional training. Exp Brain Res 2008;188:317-22.

13. Kerr CE, Sacchet MD, Lazar SW, et al. Mindfulness starts with the body: somatosensory attention and top-down modulation of cortical alpha rhythms in mindfulness meditation. Front Hum Neurosci 2013;7:12.

14. Farb N, Daubenmier J, Price CJ, et al. Interoception, contemplative practice, and health. Front Psychol 2015;6:763.

15. Xu D, Hong Y, Li J, et al. Effect of tai chi exercise on proprioception of ankle and knee joints in old people. Br J Sports Med 2004;38:50-4.

16. Cavegn EI, Riskowski JL. The effects of Tai Chi on peripheral somatosensation, balance, and fitness in hispanic older adults with type 2 diabetes: a pilot and feasibility study. Evid Based Complement Alternat Med 2015;2015:767213.

17. Mehling WE, Price C, Daubenmier JJ, et al. The multidimensional assessment of interoceptive awareness (MAIA). PLoS One 2012; $7: e 48230$

18. Sherman KJ, Wellman RD, Cook AJ, et al. Mediators of yoga and stretching for chronic low back pain. Evid Based Complement Alternat Med 2013;2013:130818.

19. Lauche R, Stumpe C, Fehr J, et al. The effects of Tai Chi and neck exercises in the treatment of chronic non-specific neck pain: a randomized controlled trial. I Pain 2016;17:1013-27.
20. Chia M, Li J. The Inner Structure of Tai Chi: Mastering the Classic Forms of Tai Chi Kunged. Vermont: Destiny Books Rochester; 2005

21. Pfingsten M, Nagel B, Emrich O, et al. Deutscher SchmerzFragebogen. Handbuch ed, Deutsche Schmerzgesellschaft e.V., 2012. Available at: http://www.dgss.org/fileadmin/pdf/12_DSF_ Manual_2012.2.pdf.

22. Korb J, Pfingsten M. Der deutsche Schmerzfragebogen-implementierte Psychometrie. Schmerz 2003;17:S47.

23. Zigmond AS, Snaith RP. The Hospital Anxiety and Depression Scale. Acta Psychiatr Scand 1983;67:361-70.

24. Herrmann C, Buss U, Snaith RP. HADS-D: Hospital Anxiety and Depression Scale-Deutsche Version. Testdokumentation und Handanweisunged. Bern: Huber; 1993.

25. Cohen S, Kamarck T, Mermelstein R. A global measure of perceived stress. J Health Soc Behav 1983;24:385-439.

26. Büssing A, Recchia DR. Spiritual and non-spiritual needs among German soldiers and their relation to stress perception, PTDS symptoms, and life satisfaction: results from a structural equation modeling approach. J Relig Health 2015;55:747-64.

27. Krippendorff K. Content Analysis: An Introduction to Its Methodology. Thousand Oaks, CA: Sage; 2004.

28. Michaelson P, Sjolander P, Johansson H. Factors predicting pain reduction in chronic back and neck pain after multimodal treatment. Clin I Pain 2004;20:447-54.

29. Cramer H, Lauche R, Hohmann C, et al. Yoga for chronic neck pain: a 12-month follow-up. Pain Med 2013;14:541-8.

30. McWilliams LA, Cox BJ, Enns MW. Mood and anxiety disorders associated with chronic pain: an examination in a nationally representative sample. Pain 2003;106:127-33.

31. Bair MJ, Poleshuck EL, Wu J, et al. Anxiety but not social stressors predict 12-month depression and pain severity. Clin J Pain 2013;29:95-101.

32. Linton SJ. A review of psychological risk factors in back and neck pain. Spine (Phila Pa 1976) 2000;25:1148-56.

33. Linton SJ, Gotestam KG. Relations between pain, anxiety, mood and muscle tension in chronic pain patients. A correlation study. Psychother Psychosom 1985;43:90-5.

34. Herrmann C. International experiences with the Hospital Anxiety and Depression Scale-a review of validation data and clinical results. J Psychosom Res 1997;42:17-41.

35. Naito E, Morita T, Amemiya K. Body representations in the human brain revealed by kinesthetic illusions and their essential contributions to motor control and corporeal awareness. Neurosci Res 2016;104:16-30.

36. Nedeljkovic M, Burgler C, Wirtz PH, et al. Getting started with taiji: investigating students expectations and teachers appraisals of taiji beginners courses. Evid Based Complement Alternat Med 2012;2012:595710.

37. Schitter AM, Ausfeld-Hafter B, Nedeljkovic M. Investigating short- and long-term transfer effects of a Taiji beginner course in participants' daily life. J Integr Med 2013;11:295-304.

38. Hackney ME, Wolf SL. Impact of Tai Chi Chu'an practice on balance and mobility in older adults: an integrative review of 20 years of research. J Geriatr Phys Ther 2014;37:127-35.

39. Tse SK, Bailey DM. T'ai chi and postural control in the well elderly. Am J Occup Ther 1992;46:295-300.

40. Chen WW, Sun WY. Tai chi chuan, an alternative form of exercise for health promotion and disease prevention for older adults in the community. Int O Community Health Educ 1996;16:333-9.

41. Forest WR. Anticipatory postural adjustment and Tai Chi Chuan. Biomed Sci Instrum 1997;33:65-70.

42. Jacobson BH, Chen HC, Cashel C, et al. The effect of T'ai Chi Chuan training on balance, kinesthetic sense, and strength. Percept Mot Skills 1997;84:27-33.

43. Gyllensten AL, Hui-Chan CW, Tsang WW. Stability limits, singleleg jump, and body awareness in older Tai Chi practitioners. Arch Phys Med Rehabil 2010;91:215-20.

44. Cramer H, Lauche R, Hohmann C, et al. Randomized-controlled trial comparing yoga and home-based exercise for chronic neck pain. Clin J Pain 2013;29:216-23.

45. Ikai S, Uchida H, Suzuki T, et al. Effects of yoga therapy on postural stability in patients with schizophrenia-spectrum 
disorders: a single-blind randomized controlled trial. J Psychiatr Res 2013;47:1744-50.

46. Kelley KK, Aaron D, Hynds K, et al. The effects of a therapeutic yoga program on postural control, mobility, and gait speed in community-dwelling older adults. J Altern Complement Med 2014;20:949-54.
47. Wayne PM, Kaptchuk TJ. Challenges inherent to t'ai chi research: part I-t'ai chi as a complex multicomponent intervention. J Altern Complement Med 2008;14:95-102.

48. Wayne PM, Kaptchuk TJ. Challenges inherent to t'ai chi research: part II-defining the intervention and optimal study design. J Altern Complement Med 2008;14:191-7. 